\title{
Heterosis for Productivity and Fibre Quality Traits among Hybrids Derived from Diverse Lines of Gossypium hirsutum L.
}

\author{
M.J. Pavitra*, S.T. Kajjidoni and Venkatesh \\ Department of Genetics and Plant Breeding, College of Agriculture, Dharwad \\ University of Agricultural Sciences, Dharwad - 580 005, Karnataka, India \\ *Corresponding author
}

\section{Keywords}

Cotton, Ginning outturn, Heterosis, Intraspecific, Line $x$ Tester analysis

Article Info

Accepted:

12 January 2019

Available Online:

10 February 2019

\section{A B S T R A C T}

A study on heterosis for seed cotton yield and fibre quality traits was carried out at Agricultural Research Station, Dharwad farm during kharif 2017. The material comprised of 28 intraspecific hybrids, their parents and standard check DHH-11 which were evaluated in randomized complete block design (RCBD) with two replications. The analysis of variance indicated the presence of substantial variability among the experimental materials for yield and fibre quality traits. The hybrid KDHH-22 exhibited significant positive heterosis over the standard check for seed cotton yield per plant and ginning outturn. The hybrid KDHH-4 recorded high significant standard heterosis for number of sympodia per plant and uniformity ratio, as well as high mean performance for plant height and lint index. The other hybrid, KDHH-11 exhibited significant standard heterosis for seed index and fibre strength. The hybrids KDHH-9, KDHH-24, KDHH-19, KDHH-27 exhibited high significant the standard heterosis for number of monopodia per plant, number of bolls per plant, boll weight and micronaire value respectively in desirable direction. The cross KDHH-13 recorded significant heterosis over standard check for UHML and elongation. The hybrids KDHH-22, KDHH-1, KDHH-8, KDHH-7 and KDHH- 6 were identified as best hybrids for seed cotton yield and fibre quality traits which can be exploited through heterosis breeding.

\section{Introduction}

Cotton, the king of fibre is one of the momentous and important cash crops exercising a profound influence on economics and social affairs of the world.

In India, the crop is being grown in an area of 12.3 million hectares, producing 28.50 million bales with a productivity of $504 \mathrm{~kg}$ $\mathrm{ha}^{-1}$. In Karnataka, cotton is being grown in an area of 5.65 lakh hectares with production and productivity of 19.0 lakh bales and 572 $\mathrm{kg} \mathrm{ha}^{-1}$ respectively (Anon., 2018).

In the present study, an effort was made to evaluate the utility of selected diversified new parental lines for exploiting the hybrid vigour for seed cotton yield and fibre quality traits. 


\section{Materials and Methods}

The present investigation on the evaluation of intraspecific hybrids for seed cotton yield and fibre quality traits was conducted during kharif 2017 at Agricultural Research Station, Dharwad. Experimental material comprised of 14 female parents viz., RAH-803-5, RAH803-6, Surabhi selection-36, HET Group line5, HET Group line-15, HET Group line-19, HET Group line-21, HET Group line-47, DRGR-178-4-14, DRGR-178-4-15, Suraj-44, DRGR-4-63, DRGR-178-109 and (DSC-7 $\times$ DSC-68)-121 identified based on plant type traits in combination with two male parents (ARBH-813 and Surabhi) and one standard check DHH-11. The lines were selected from the different backgrounds viz., compact, robust, RGR and stay green plant types. The 28 hybrids along with 16 parents and a standard check were raised during kharif 2017 in two replications in a randomized complete block design (RCBD). The per cent heterosis of $\mathrm{F}_{1}$ over their mid parent (MP), better parent (BP) and standard check (SC) were computed as per the method suggested by Turner (1953) and Hayes et al., (1955).

\section{Results and Discussion}

The analysis of variance indicated the presence of substantial variability for yield and fibre quality traits. The range of heterosis over better parent and the standard check is presented in Table 1. The better parent heterosis for number of monopodia recorded high range of -41.67 to 216.67 per cent followed by seed cotton yield per plant (3.34 to $208.84 \%$ ) and number of bolls per plant (24.69 to 156.33). Seed cotton yield per plant exhibited high range of standard heterosis $(-31.81$ to $57.59 \%)$ followed by fibre elongation (-16.28 to $51.16 \%)$, boll weight (1.84 to $47.74 \%)$ and the number of bolls per plant (-35.46 to $43.62 \%)$. Heterosis over the standard check for different quantitative characters in intraspecific cotton hybrids and mean performance of the top five hybrids identified based on seed cotton yield and their corresponding quantitative traits are presented in Table 1 and 2. For seed cotton yield per plant, the hybrid KDHH-22 (57.59 \%), KDHH-1 (43.45 \%) and KDHH-7 (37.87 \%) exhibited high standard heterosis. Most of these high heterotic cross combinations involve either HET Group line-47 or RAH 803-5 as a female parent. Thus, these three high heterotic hybrids can be used to exploit heterotic values for seed cotton yield per plant. Similar results were reported by Sawarkar et al., (2015), Chhavikant et al., (2017) and Monicashree et al., (2017). High significant positive heterosis over the standard check (DHH-11) for the number of bolls per plant was exhibited by five crosses. Abro et al., (2014), Sawarkar et al., (2015), Chhavikant et al., (2017) and Monicashree et al., (2017) also reported similar results. For boll weight, hybrids KDHH-19 (47.74 \%), KDHH-18 (44.35 \%) and KDHH-11 (37.57 $\%)$ showed high significant positive standard heterosis. A similar report was also made by Chhavikant et al., (2017). For trait number of monopodia per plant, the cross KDHH-9 recorded the highest significant negative heterosis over standard check DHH-11. The similar trend was evident from the study Chhavikant et al., (2017). The hybrids KDHH-4 and KDHH-12 exhibited high significant positive heterosis for the number of sympodia per plant. Similar findings were reported by Abro et al., (2014) and Chhavikant et al., (2017). The hybrids KDHH-11 (19.05\%) and KDHH-19 (19.05 $\%$ ) exhibited highest maximum heterosis over the check for seed index. The high level of heterosis for seed cotton yield per plant recorded by the crosses viz., KDHH-22, KDHH-1, KDHH-8 and KDHH-7 which can be mainly attributed to the cumulative action of component traits such as number of sympodia per plant, number of bolls per plant and boll weight. 
Table.1 Range of heterosis over better parent and standard check and mean performance of top five hybrids identified based on seed cotton yield and their corresponding quantitative traits

\begin{tabular}{|c|c|c|c|c|c|c|c|c|c|}
\hline $\begin{array}{l}\text { Sl. } \\
\text { No. }\end{array}$ & Characters & $\begin{array}{l}\text { Better } \\
\text { parent } \\
(\%)\end{array}$ & $\begin{array}{c}\text { Standard } \\
\text { heterosis } \\
(\%)\end{array}$ & $\begin{array}{c}\text { KDHH-22 } \\
\text { (HET Group lines- } \\
47 \times \text { Surabhi) }\end{array}$ & $\begin{array}{c}\text { KDHH-1 } \\
\text { (RAH-803-5 x } \\
\text { ARBH-813) }\end{array}$ & $\begin{array}{c}\text { KDHH-8 } \\
\text { (HET Group lines- } \\
47 \times \text { ARBH-813) }\end{array}$ & $\begin{array}{c}\text { KDHH-7 } \\
\text { (HET Group lines- } \\
21 \times \text { ARBH-813) }\end{array}$ & $\begin{array}{c}\text { KDHH-6 } \\
\text { (HET Group lines- } \\
19 \times \text { ARBH-813) }\end{array}$ & $\begin{array}{c}\text { Standard } \\
\text { check } \\
\text { DHH-11 }\end{array}$ \\
\hline 1 & Plant height $(\mathrm{cm})$ & $\begin{array}{c}-17.09 \text { to } \\
16.83\end{array}$ & $\begin{array}{c}-13.19 \text { to } \\
8.73\end{array}$ & 99.00 & 105.00 & 112.50 & 106.20 & 111.40 & 112.2 \\
\hline 2 & $\begin{array}{l}\text { Number of } \\
\text { monopodia }\end{array}$ & $\begin{array}{c}-41.67 \text { to } \\
216.67\end{array}$ & $\begin{array}{c}-63.16 \text { to } \\
36.84\end{array}$ & 1.9 & 1.3 & 1.7 & 1.3 & 1.9 & 1.9 \\
\hline 3 & $\begin{array}{l}\text { Number of } \\
\text { sympodia }\end{array}$ & $\begin{array}{l}-2.01 \text { to } \\
23.24\end{array}$ & $\begin{array}{c}-11.52 \text { to } \\
11.52\end{array}$ & 15.7 & 15 & 17.8 & 16.7 & 17.9 & 16.5 \\
\hline 4 & $\begin{array}{l}\text { Days to } 50 \text { per } \\
\text { cent flowering }\end{array}$ & $\begin{array}{c}-9.09 \text { to } \\
0.00\end{array}$ & $\begin{array}{c}-5.17 \text { to } \\
6.90\end{array}$ & 57.50 & 59.50 & 58.00 & 56.50 & 58.00 & 59.00 \\
\hline 5 & $\begin{array}{l}\text { Number of bolls } \\
\text { per plant }\end{array}$ & $\begin{array}{c}24.69 \text { to } \\
156.33\end{array}$ & $\begin{array}{c}-35.46 \text { to } \\
43.62\end{array}$ & 20.25 & 13.05 & 14.00 & 18.25 & 12.30 & 14.1 \\
\hline 6 & Boll weight(g) & $\begin{array}{l}-6.64 \text { to } \\
30.31\end{array}$ & $\begin{array}{l}1.84 \text { to } \\
47.74\end{array}$ & 4.07 & 3.99 & 4.43 & 4.15 & 4.06 & 3.54 \\
\hline 7 & $\begin{array}{l}\text { Seed cotton yield } \\
\text { per plant }(\mathrm{g})\end{array}$ & $\begin{array}{l}3.34 \text { to } \\
208.84\end{array}$ & $\begin{array}{c}-31.81 \text { to } \\
57.59\end{array}$ & 74.73 & 67.5 & 60.32 & 65.11 & 54.74 & 47.23 \\
\hline 8 & $\begin{array}{l}\text { Ginning outturn } \\
(\%)\end{array}$ & $\begin{array}{c}-11.69 \text { to } \\
3.07\end{array}$ & $\begin{array}{l}-8.77 \text { to } \\
2.99\end{array}$ & 37.93 & 36.80 & 36.30 & 33.91 & 36.00 & 36.83 \\
\hline 9 & Seed index (g) & $\begin{array}{l}-24 \text { to } \\
13.64\end{array}$ & $\begin{array}{c}-14.29 \text { to } \\
19.05\end{array}$ & 11.00 & 9.00 & 11.00 & 10.50 & 10.50 & 10.5 \\
\hline 10 & Lint index (g) & $\begin{array}{c}-21.65 \text { to } \\
15.19\end{array}$ & $\begin{array}{c}-20.10 \text { to } \\
13.24\end{array}$ & 6.71 & 5.23 & 6.28 & 5.38 & 5.89 & 6.12 \\
\hline 11 & UHML (mm) & $\begin{array}{c}-16.12 \text { to } \\
5.40\end{array}$ & $\begin{array}{c}-3.62 \text { to } \\
17.03\end{array}$ & 28.00 & 27.60 & 28.80 & 28.80 & 29.70 & 27.6 \\
\hline 12 & $\begin{array}{l}\text { Fibre strength } \\
\left(\mathrm{g} \mathrm{tex}^{-1}\right)\end{array}$ & $\begin{array}{c}-7.91 \text { to } \\
6.59\end{array}$ & $\begin{array}{c}0.39 \text { to } \\
12.55\end{array}$ & 26.90 & 25.80 & 26.6 & 27.10 & 27.10 & 25.5 \\
\hline 13 & $\begin{array}{l}\text { Micronaire } \\
\left(\mu \mathrm{g} \text { inch }^{-1}\right)\end{array}$ & $\begin{array}{c}-15.49 \text { to } \\
1.85\end{array}$ & $\begin{array}{l}-16.40 \text { to } \\
1.82\end{array}$ & 4.40 & 4.08 & 4.34 & 3.93 & 4.20 & 4.36 \\
\hline 14 & $\begin{array}{l}\text { Uniformity ratio } \\
(\%)\end{array}$ & $\begin{array}{c}-3.52 \text { to } \\
6.44\end{array}$ & $\begin{array}{l}-1.08 \text { to } \\
5.99\end{array}$ & 86.00 & 82.60 & 85.60 & 87.70 & 85.30 & 83.5 \\
\hline 15 & $\begin{array}{l}\text { Fibre elongation } \\
(\%)\end{array}$ & $\begin{array}{c}-32.81 \text { to } \\
36.96\end{array}$ & $\begin{array}{c}-16.28 \text { to } \\
51.16\end{array}$ & 4.30 & 4.50 & 5.20 & 5.90 & 5.50 & 4.30 \\
\hline
\end{tabular}


Table.2 Heterosis over standard check for different quantitative characters in intraspecific cotton hybrids

\begin{tabular}{|c|c|c|c|c|c|c|c|c|}
\hline $\begin{array}{l}\text { Sl. } \\
\text { No }\end{array}$ & Crosses & $\begin{array}{c}\text { Plant } \\
\text { height } \\
(\mathbf{c m})\end{array}$ & $\begin{array}{l}\text { Number of } \\
\text { monopodi } \\
\text { a per plant }\end{array}$ & $\begin{array}{c}\begin{array}{c}\text { Number } \\
\text { of }\end{array} \\
\text { sympodia } \\
\text { per plant }\end{array}$ & $\begin{array}{c}\text { Days to } \\
50 \text { per } \\
\text { cent } \\
\text { flowering }\end{array}$ & $\begin{array}{c}\text { Number } \\
\text { of bolls } \\
\text { per plant }\end{array}$ & $\begin{array}{c}\text { Boll } \\
\text { weight } \\
\text { (g) }\end{array}$ & $\begin{array}{c}\text { Seed } \\
\text { cotton } \\
\text { yield per } \\
\text { plant }(\mathrm{g})\end{array}$ \\
\hline 1 & KDHH-1 & -6.42 & -31.58 & -9.09 & 2.59 & -7.45 & 12.71 & $43.45 * *$ \\
\hline 2 & KDHH-2 & 3.68 & -13.16 & -9.09 & $6.90 *$ & $-21.99 * *$ & 9.60 & $22.06^{* *}$ \\
\hline 3 & KDHH-3 & -4.81 & $-52.63^{*}$ & -0.61 & -1.72 & $-25.53 * *$ & $24.15^{*}$ & $-31.81 * *$ \\
\hline 4 & KDHH-4 & 7.04 & -15.79 & $11.52 *$ & 0.86 & $-35.46^{* *}$ & $35.59 * *$ & $-4.51^{*}$ \\
\hline 5 & KDHH-5 & -7.13 & -36.84 & -4.24 & -5.17 & $-14.89^{*}$ & $35.73 * *$ & -3.35 \\
\hline 6 & KDHH-6 & -0.71 & $-52.63^{*}$ & 8.48 & 0.00 & $-12.77^{*}$ & 14.69 & $15.90^{* *}$ \\
\hline 7 & KDHH-7 & -5.35 & -31.58 & 1.21 & 2.59 & $29.43 * *$ & 17.23 & $37.87 * *$ \\
\hline 8 & KDHH-8 & -0.27 & -10.53 & 7.88 & 0.00 & -0.71 & $25.28^{*}$ & $27.73 * *$ \\
\hline 9 & KDHH-9 & -11.23 & $-63.16^{* *}$ & -7.88 & -3.45 & 3.90 & 20.20 & $-8.43 * *$ \\
\hline 10 & KDHH-10 & 1.34 & $-42.11^{*}$ & 5.45 & 0.00 & -4.61 & $29.94 *$ & $11.18^{* *}$ \\
\hline 11 & KDHH-11 & 5.08 & -21.05 & -6.06 & -3.45 & $-20.57 * *$ & $37.57 * *$ & $13.96 * *$ \\
\hline 12 & KDHH-12 & -3.83 & $-42.11 *$ & $9.70^{*}$ & -0.86 & 4.96 & $30.65^{*}$ & $23.88^{* *}$ \\
\hline 13 & KDHH-13 & -1.34 & -31.58 & -0.61 & 0.00 & $-28.37 * *$ & $32.77^{*}$ & $-21.73^{* *}$ \\
\hline 14 & KDHH-14 & 5.64 & -26.32 & 3.03 & 0.86 & $-17.73^{* *}$ & 20.90 & $4.86^{*}$ \\
\hline 15 & KDHH-15 & -11.76 & -26.32 & -3.03 & 0.86 & $13.48 *$ & 3.81 & $-12.45^{* *}$ \\
\hline 16 & KDHH-16 & $-12.75^{*}$ & -15.79 & 6.06 & $6.90^{*}$ & 0.71 & 18.64 & $-29.94 * *$ \\
\hline 17 & KDHH-17 & 7.04 & 10.53 & 7.27 & 2.59 & 6.38 & 19.63 & $-4.64 *$ \\
\hline 18 & KDHH-18 & 1.43 & -31.58 & 6.06 & 3.45 & $-27.66^{* *}$ & $44.35 * *$ & $-19.39 * *$ \\
\hline 19 & KDHH-19 & -2.50 & -21.05 & -11.52 & 0.00 & $-26.24 * *$ & $47.74 * *$ & $-18.80 * *$ \\
\hline 20 & KDHH-20 & 8.73 & -26.32 & -0.30 & 0.86 & 8.16 & 3.95 & $-8.53^{* *}$ \\
\hline 21 & KDHH-21 & -3.92 & -26.32 & 0.61 & 0.86 & $21.28 * *$ & $29.52 *$ & -1.55 \\
\hline 22 & KDHH-22 & -11.76 & 0.00 & -4.85 & -0.86 & $43.62 * *$ & 14.97 & $57.59 * *$ \\
\hline 23 & KDHH-23 & -8.20 & $-52.63^{*}$ & -1.21 & 4.31 & 6.38 & 15.25 & -3.02 \\
\hline 24 & KDHH-24 & $-13.19 *$ & -31.58 & -7.27 & 3.45 & $33.33 * *$ & 17.51 & $12.75^{* *}$ \\
\hline 25 & KDHH-25 & -3.48 & -21.05 & -3.64 & 0.86 & -8.51 & $30.79^{*}$ & $-9.18 * *$ \\
\hline 26 & KDHH-26 & 5.44 & $-47.37 *$ & 5.45 & 4.31 & 0.71 & $33.47 * *$ & -0.86 \\
\hline 27 & KDHH-27 & -8.82 & -31.58 & $-9.70^{*}$ & 0.86 & -6.38 & $25.71 *$ & $-5.57 * *$ \\
\hline \multirow[t]{4}{*}{28} & KDHH-28 & 4.55 & 36.84 & 0.00 & 1.72 & -2.84 & 1.84 & 1.27 \\
\hline & Mean & 107.67 & 1.20 & 15.96 & 59.18 & 11.64 & 4.23 & 41.75 \\
\hline & Range & $\begin{array}{l}97.4- \\
120.1\end{array}$ & $0.7-2.1$ & $14.6-18.4$ & $55-62$ & $\begin{array}{l}9.10- \\
20.25\end{array}$ & $\begin{array}{c}3.60- \\
5.23\end{array}$ & $\begin{array}{l}32.51- \\
74.43\end{array}$ \\
\hline & $\begin{array}{l}\text { C. D. at } 5 \\
\%\end{array}$ & 13.67 & 0.72 & 1.59 & 3.60 & 1.70 & 0.74 & 2.10 \\
\hline
\end{tabular}


Table 2. Contd...

\begin{tabular}{|c|c|c|c|c|c|c|c|c|c|}
\hline $\begin{array}{l}\text { Sl. } \\
\text { No }\end{array}$ & Crosses & $\begin{array}{c}\text { Ginning } \\
\text { outturn } \\
(\%)\end{array}$ & $\begin{array}{l}\text { Seed } \\
\text { index } \\
(\mathrm{g})\end{array}$ & $\begin{array}{c}\text { Lint } \\
\text { index }\end{array}$ & $\begin{array}{l}\text { UHML } \\
(\mathbf{m m})\end{array}$ & $\begin{array}{c}\text { Fibre } \\
\text { strength } \\
\left(\mathrm{g} \mathrm{tex}^{-1}\right)\end{array}$ & $\begin{array}{l}\text { Micronaire } \\
\left(\mu \text { inch }^{-1}\right)\end{array}$ & $\begin{array}{c}\text { Uniformity } \\
\text { ratio }(\%)\end{array}$ & $\begin{array}{c}\text { Elongation } \\
(\%)\end{array}$ \\
\hline 1 & KDHH-1 & -0.08 & $-14.29 *$ & -14.54 & 0.00 & 1.18 & $-7.06^{* *}$ & -1.08 & $4.65^{* *}$ \\
\hline 2 & KDHH-2 & $-4.97 *$ & -4.76 & -12.17 & 1.09 & $5.10 * *$ & $-4.56 * *$ & 2.40 & $16.28^{* *}$ \\
\hline 3 & KDHH-3 & -2.25 & 4.76 & 1.14 & 5.80 & $3.73^{*}$ & -0.91 & $5.51 * *$ & $34.88 * *$ \\
\hline 4 & KDHH-4 & -1.95 & $14.29 *$ & 10.70 & $9.06^{* *}$ & $4.71 * *$ & $-7.29 * *$ & $5.99 * *$ & $46.51 * *$ \\
\hline 5 & KDHH-5 & $-4.43 *$ & 4.76 & -2.37 & $12.68 * *$ & $5.88 * *$ & $-3.64 * *$ & 3.11 & $37.21^{* *}$ \\
\hline 6 & KDHH-6 & -2.25 & 0.00 & -3.68 & $7.61^{*}$ & $6.27 * *$ & $-4.33^{* *}$ & 2.16 & $27.91 * *$ \\
\hline 7 & KDHH-7 & $-7.93 * *$ & 0.00 & -12.17 & 4.35 & $6.27 * *$ & $-10.48 * *$ & $5.03 * *$ & $37.21 * *$ \\
\hline 8 & KDHH-8 & -1.44 & 4.76 & 2.61 & 4.35 & $4.31 * *$ & -1.14 & 2.51 & $20.93 * *$ \\
\hline 9 & KDHH-9 & -2.25 & 4.76 & 0.90 & $8.70^{*}$ & $6.27 * *$ & $-10.25^{* *}$ & 3.11 & $39.53^{* *}$ \\
\hline 10 & KDHH-10 & -2.25 & 4.76 & 1.14 & $11.59 * *$ & $6.67 * *$ & $-12.76^{* *}$ & $5.93 * *$ & $46.51^{* *}$ \\
\hline 11 & KDHH-11 & $-6.60 * *$ & $19.05^{*}$ & 6.94 & $8.70^{*}$ & $12.55^{* *}$ & $-15.49 * *$ & 0.00 & $25.58^{* *}$ \\
\hline 12 & KDHH-12 & -0.08 & 4.76 & 4.74 & $7.97^{*}$ & $6.67 * *$ & -0.23 & $4.79 * *$ & $34.88^{* *}$ \\
\hline 13 & KDHH-13 & -2.25 & 4.76 & 1.14 & $17.03 * *$ & $9.80 * *$ & $-7.06^{* *}$ & 2.99 & $51.16^{* *}$ \\
\hline 14 & KDHH-14 & 2.09 & -9.52 & -6.70 & 1.09 & $7.84 * *$ & $-13.90 * *$ & 1.08 & $-16.28 * *$ \\
\hline 15 & KDHH-15 & -0.52 & -4.76 & -3.59 & 2.17 & $5.49 * *$ & $-4.33^{* *}$ & 1.56 & $-6.98 * *$ \\
\hline 16 & KDHH-16 & $-4.43^{*}$ & $-14.29 *$ & $-20.10^{*}$ & 3.99 & $9.02 * *$ & $-9.34 * *$ & $4.91 * *$ & $30.23^{* *}$ \\
\hline 17 & KDHH-17 & -0.08 & -9.52 & -9.80 & -3.62 & 0.78 & -1.37 & 1.56 & 0.00 \\
\hline 18 & KDHH-18 & -2.25 & 4.76 & 1.14 & 5.43 & $7.06^{* *}$ & -1.82 & $3.71 *$ & $27.91 * *$ \\
\hline 19 & KDHH-19 & $-8.77 * *$ & $19.05^{*}$ & 3.19 & $11.59 * *$ & $7.06^{* *}$ & $-5.47 * *$ & $4.79 * *$ & $46.51^{* * *}$ \\
\hline 20 & KDHH-20 & $-4.43^{*}$ & 0.00 & -6.94 & 7.97* & $7.45^{* *}$ & $-5.47 * *$ & 2.28 & $37.21 * *$ \\
\hline 21 & KDHH-21 & -0.08 & 4.76 & 9.40 & $6.88^{*}$ & $8.24 * *$ & $-3.42 *$ & 2.87 & $32.56^{* *}$ \\
\hline 22 & KDHH-22 & 2.99 & 4.76 & 9.64 & 1.45 & $5.49 * *$ & 0.23 & 2.99 & 0.00 \\
\hline 23 & KDHH-23 & -0.08 & -4.76 & -4.82 & 4.35 & $9.41 * *$ & $-11.16^{* *}$ & 3.23 & $20.93^{* *}$ \\
\hline 24 & KDHH-24 & $-8.77 * *$ & 0.00 & -13.32 & $9.78 * *$ & $9.41 * *$ & $-11.16^{* *}$ & 1.44 & $23.26^{* *}$ \\
\hline 25 & KDHH-25 & -0.08 & 4.76 & 4.49 & 6.16 & $5.10^{* *}$ & -1.37 & 3.23 & $34.88^{* *}$ \\
\hline 26 & KDHH-26 & 2.09 & 9.52 & 13.24 & 5.43 & $10.20^{* *}$ & 1.82 & 2.75 & $27.91 * *$ \\
\hline 27 & KDHH-27 & $-4.43 *$ & 0.00 & -6.94 & 1.81 & 0.39 & $-16.40 * *$ & 1.80 & $2.33 * *$ \\
\hline \multirow[t]{4}{*}{28} & KDHH-28 & 2.09 & -4.76 & -1.72 & 6.16 & $5.88 * *$ & -0.46 & 1.20 & $22.09 * *$ \\
\hline & Mean & 35.99 & 10.67 & 6.01 & 29.27 & 26.69 & 4.12 & 85.85 & 5.38 \\
\hline & Range & $\begin{array}{c}33.60- \\
37.93\end{array}$ & $\begin{array}{l}9.0- \\
12.5\end{array}$ & $\begin{array}{c}4.89- \\
6.93\end{array}$ & $\begin{array}{c}26.60- \\
32.30\end{array}$ & $\begin{array}{c}25.60- \\
28.70\end{array}$ & $3.67-4.47$ & $82.3-88.5$ & $4-6.514$ \\
\hline & C. D. at $5 \%$ & 1.37 & 1.49 & 1.03 & 1.77 & 0.78 & 0.05 & 1.40 & 0.04 \\
\hline
\end{tabular}

Among 28 intrahirsutum hybrids, KDHH-13 (17.03\%) exhibited the highest significant positive standard heterosis for UHML. A total of 12 hybrids recorded significant positive heterosis over standard check DHH-11. Abro et al., (2014) and Sawarkar et al., (2015) reported similar results. The other important fibre quality parameter is fibre strength and it plays a crucial role in the textile industry. The hybrid KDHH$11(12.55 \%)$ exhibited high significant positive heterosis over the standard check followed by KDHH-26 (10.20\%) and KDHH-13 (9.80\%) for fibre strength. Sawarkar et al., (2015) observed significant positive heterosis for fibre strength. Medium micronare value is always preferred for textile machine. For this trait, the 
hybrid KDHH-27 exhibited high significant negative heterosis over standard check. A total of 19 crosses recorded significant negative heterosis over standard check DHH-11. The significant negative heterosis over standard check was reported by Sawarkar et al., (2015) and Monicashree et al., (2017). Among 28 hybrids, eight hybrids showed significant positive heterosis over DHH-11 for uniformity ratio. The hybrid $\mathrm{KDHH}-4$ had high significant positive heterosis over standard check DHH-11 for uniformity ratio. These findings are in accordance with Monicashree et al., (2017). For fibre elongation, the hybrid $\mathrm{KDHH}-13$ exhibited significant positive heterosis over standard check DHH-11. Superior hybrids KDHH-22, KDHH-1, KDHH-8, KDHH-7 and KDHH-6 recorded high per se performance over standard check DHH-11 for seed cotton yield per plant, boll weight, number of bolls per plant, seed index, UHML and fibre strength.

The present investigation paves way for exposing new hybrids that could be exploited through heterosis breeding to produce elite hybrids which can outperform the present hybrids in terms of seed cotton yield. The hybrid KDHH-22 exhibited the highest per se performance with significant heterosis over better parent and standard check DHH-11 for the majority of yield components followed by KDHH-1, KDHH-8, KDHH-7 and KDHH-6 hybrids. The hybrids identified for high heterosis for seed cotton yield also exhibited significant standard heterosis for most of fibre quality traits. The high heterotic response in these hybrids was due to positive heterosis for yield contributing characters like number of bolls, boll weight and ginning outturn. It is suggested to evaluate these hybrids in large scale trial to confirm their potentiality for future exploitation at the commercial level.

\section{References}

Abro, S., Laghari, S., Deho, Z. A. and Manjh, M. A., 2014, To estimates heterosis and heterobeltosis of yield and quality traits in upland cotton. J. Biol. Agric. Healthcare., 4: 2224-3208.

Anonymous, 2018, Annu. Rep. ICAR- All India Coordinated Research Project on cotton, pp. 2-5.

Chhavikant, M., Nirania, K. S., Kumar, A. and Pundir, S. R., 2017, Heterosis studies for seed cotton yield and other traits in upland cotton (Gossypium hirsutum L.). J. Pharmacogn. Phytochem., 6(6): 583-586.

Hayes, H. K., Immer, I. R. and Smith, D. C., 1955, Methods Plant Breeding. McGraw Hill Book Company Inc., New York. pp. 51-56.

Monicashree, C., Balu, A. P. and Gunasekaran, M., 2017, Combining ability and heterosis studies on yield and fibre quality traits in upland cotton. Int. J. Curr. Microbiol. Appl. Sci., 6(8): 912-927.

Panse, V. G. and Sukhatme, P. V., 1978, Statistical methods for Agricultural workers, $2^{\text {nd }}$ Edn., Indian Council of Agricultural Research, New Delhi, (India), p. 124.

Sawarkar, M., Solanke, A., Mhasal, G. S. and Deshmukh, S. B., 2015, Combining ability analysis and heterosis for seed cotton yield, its components and quality traits in Gossypium hirsutum L. Indian J. Agric. Res., 49(2): 154-159.

Turner, J. R., 1953, A study of heterosis in upland cotton. II. Combining ability and inbreeding effects. Agron. J., 43: 487490.

\section{How to cite this article:}

Pavitra, M.J., S.T. Kajjidoni and Venkatesh. 2019. Heterosis for Productivity and Fibre Quality Traits among Hybrids Derived from Diverse Lines of Gossypium hirsutum L. Int.J.Curr.Microbiol.App.Sci. 8(02): 1379-1384. doi: https://doi.org/10.20546/ijcmas.2019.802.162 